\title{
Green-function method for a mixed-valence Hamiltonian
}

\author{
Acirete S. Rosa Simões and J. R. Iglesias \\ Instituto de Física, Universidade Federal do Rio Grande do Sul, 90000 Porto Alegre, Rio Grande do Sul, Brazil
}

\author{
E. V. Anda
}

Instituto de Física, Universidade Federal Fluminense, 24000 Niterói, Rio de Janeiro, Brazil

(Received 30 December 1982; revised manuscript received 25 October 1983)

\begin{abstract}
A Green-function diagrammatic method is utilized to calculate the occupation number of a narrow $(f)$ band in a mixed-valence system. The method is a generalization of the Hubbard-I approximation for a two-band Hamiltonian. The results at $T=0 \mathrm{~K}$ indicate the existence of an intermediate-valence state for finite hybridization between the bands, and abrupt transitions for values of $G$ comparable to the half bandwidth of the conduction band. A metal-insulator transition is also obtained.
\end{abstract}

\section{INTRODUCTION}

It seems well established that a two-hybridized-band model is able to describe the electronic properties of intermediate valence systems. ${ }^{1}$. From the original work of Ramirez et al., ${ }^{2}$ who explained the $\gamma-\alpha$ transition of metallic cerium, the idea that the valence transition is due to the raising of the $4 f$ level through the Fermi energy has been explored in many ways by several authors. ${ }^{3-12}$

The effect of the hybridization, neglected in the initial model, ${ }^{1,2}$ was taken into account in the form of a virtual bound state $\mathrm{s}^{3-6}$ or as a coherent hybridization between the $f$ and conduction bands. ${ }^{9-12}$ Also, both the Coulomb $f-f$ $(U)$ and $f$-conduction-electron $(G)$ interactions were treated by different schemes, the existence of an abrupt transition driven by the later interaction, $G$, being a controversial point. Actually, when $G$ is treated in the mean-field approximation $^{1,2,5,11}$ an abrupt transition between two valence states can occur. Some authors, using the alloyanalogy approximation, also obtained a sharp transition, ${ }^{10}$ while some others obtained a continuous transition ${ }^{9}$ or no transition at all. ${ }^{7,8}$ Finally, recent results ${ }^{10-12}$ indicate that no stable fractionary occupation of the $f$ shell is obtained in the metallic state.

To improve the analysis of the problem we utilize an approximate diagrammatic method recently developed ${ }^{13}$ to calculate the Green functions of the system. We present here preliminary results for $T=0 \mathrm{~K}$. Finitetemperature specific-heat computations are in progress.

\section{HAMILTONIAN AND GREEN FUNCTIONS}

Following Ref. 13, we separate the Hamiltonian into two terms: a local Hamiltonian $\mathscr{H}_{0}$, which includes all the electronic correlations and can be solved exactly, and a hopping term, $\mathscr{H}_{I}$, which takes into account the finite bandwidth of the conduction band and the hybridization contribution.

Calling $f_{i \sigma}^{\dagger}\left(d_{i \sigma}^{\dagger}\right)$ the operator that creates an electron in an $f$ (conduction) state, at the $i$ site with spin $\sigma$, the Hamiltonian reads

$$
\begin{gathered}
\mathscr{H}=\mathscr{H}_{0}+\mathscr{H}_{I}, \\
\mathscr{H}_{0}=\sum_{i, \sigma}\left[E_{0} f_{i \sigma}^{\dagger} f_{i \sigma}+E_{B} d_{i \sigma}^{\dagger} d_{i \sigma}+\frac{1}{2} U f_{i \sigma}^{\dagger} f_{i \sigma} f_{i \bar{\sigma}}^{\dagger} f_{i \bar{\sigma}}\right. \\
\left.\quad+G \sum_{\sigma^{\prime}} f_{i \sigma}^{\dagger} f_{i \sigma} d_{i \sigma^{\prime}}^{\dagger} d_{i \sigma^{\prime}}\right] \\
\mathscr{H}_{I}=\sum_{\substack{i, j, \sigma \\
i \neq j}} T_{i j} d_{i \sigma}^{\dagger} d_{j \sigma}+V \sum_{i, \sigma}\left(d_{i \sigma}^{\dagger} f_{i \sigma}+\text { h.c. }\right)
\end{gathered}
$$

The Hamiltonian $\mathscr{H}_{0}$ can be exactly solved in terms of the operators

$$
\begin{aligned}
& F_{i \sigma, \alpha \beta \gamma}=f_{i \sigma} n_{i \bar{\sigma}}^{f \alpha} n_{i \sigma}^{d \beta} n_{i \bar{\sigma}}^{d \gamma}, \\
& D_{i \sigma, \alpha \beta \gamma}=d_{i \sigma} n_{i \bar{\sigma}}^{d \alpha} n_{i \sigma}^{f \beta} n_{i \bar{\sigma}}^{f \gamma},
\end{aligned}
$$

where

$$
\begin{aligned}
& n_{i \sigma}^{f 1}=n_{i \sigma}^{f}, \\
& n_{i \sigma}^{f 2}=1-n_{i \sigma}^{f},
\end{aligned}
$$

with similar definitions for $n_{i \sigma}^{d 1(2)}$.

The explicit expressions for the Green functions of the local Hamiltonian $\mathscr{H}_{0}$ are $^{13}$

$$
\begin{aligned}
& G_{0 i}^{f \sigma}(\omega)=\sum_{\alpha, \beta, \gamma=1}^{2} \frac{\left\langle n_{i \bar{\sigma}}^{f \alpha} n_{i \sigma}^{d \beta} n_{i \bar{\sigma}}^{d \gamma}\right\rangle}{\omega-E_{\alpha \beta \gamma}^{f}} \\
& G_{0 i}^{d \sigma}(\omega)=\sum_{\alpha, \beta, \gamma=1}^{2} \frac{\left\langle n_{i \bar{\sigma}}^{d \alpha} n_{i \sigma}^{f \beta} n_{i \bar{\sigma}}^{f \gamma}\right\rangle}{\omega-E_{\alpha \beta \gamma}^{d}},
\end{aligned}
$$

with

$$
\begin{aligned}
& E_{222}^{f}=E_{0}, E_{112}^{f}=E_{121}^{f}=E_{0}+U+G, \\
& E_{221}^{f}=E_{212}^{f}=E_{0}+G, E_{211}^{f}=E_{0}+2 G, \\
& E_{122}^{f}=E_{0}+U, E_{111}^{f}=E_{0}+U+2 G, \\
& E_{222}^{d}=E_{122}^{d}=E_{B}, \\
& E_{112}^{d}=E_{121}^{d}=E_{212}^{d}=E_{221}^{d}=E_{B}+G, \\
& E_{111}^{d}=E_{211}^{d}=E_{B}+2 G .
\end{aligned}
$$


The Green functions of the full Hamiltonian are calculated by means of an approximate diagrammatic expansion method, ${ }^{13}$ and we obtain a Dyson equation:

$$
\left[\begin{array}{cc}
G_{f}^{\sigma}(\overrightarrow{\mathrm{k}}, \omega) & G_{f d}^{\sigma}(\overrightarrow{\mathrm{k}}, \omega) \\
G_{d f}^{\sigma}(\overrightarrow{\mathrm{k}}, \omega) & G_{d}^{\sigma}(\overrightarrow{\mathrm{k}}, \omega)
\end{array}\right)=\left(\begin{array}{cc}
G_{0}^{f \sigma}(\overrightarrow{\mathrm{k}}, \omega) & 0 \\
0 & G_{0}^{d \sigma}(\overrightarrow{\mathrm{k}}, \omega)
\end{array}\right]\left[1+\left(\begin{array}{cc}
0 & V \\
V & \epsilon_{\overrightarrow{\mathrm{k}}}-E_{B}
\end{array}\right]\left[\begin{array}{cc}
G_{f}^{\sigma}(\overrightarrow{\mathrm{k}}, \omega) & G_{f d}^{\sigma}(\overrightarrow{\mathrm{k}}, \omega) \\
G_{d f}^{\sigma}(\overrightarrow{\mathrm{k}}, \omega) & G_{d}^{\sigma}(\overrightarrow{\mathrm{k}}, \omega)
\end{array}\right]\right),
$$

where $\epsilon_{\overrightarrow{\mathrm{k}}}$ is the Fourier transform of $T_{i j}$ :

$$
\epsilon_{\overrightarrow{\mathrm{k}}}=\frac{1}{N} \sum_{i, j} T_{i j} e^{-i \overrightarrow{\mathrm{k}} \cdot\left(\overrightarrow{\mathrm{R}}_{i}-\overrightarrow{\mathrm{R}}_{j}\right)}
$$

The desired Green functions are obtained from Eq. (8). The results are

$$
\begin{aligned}
G_{f}^{\sigma}(\overrightarrow{\mathrm{k}}, \omega) & =\frac{\left[1-G_{0}^{d \sigma}\left(\epsilon_{\overrightarrow{\mathrm{k}}}-E_{B}\right)\right] G_{0}^{f \sigma}}{1-V^{2} G_{0}^{f \sigma} G_{0}^{d \sigma}-\left(\epsilon_{\overrightarrow{\mathrm{k}}}-E_{B}\right) G_{0}^{d \sigma}}, \\
G_{d}^{\sigma}(\overrightarrow{\mathrm{k}}, \omega) & =\frac{G_{0}^{d \sigma}}{1-V^{2} G_{0}^{d \sigma} G_{0}^{f \sigma}-\left(\epsilon_{\overrightarrow{\mathrm{k}}}-E_{B}\right) G_{0}^{d \sigma}} .
\end{aligned}
$$

It can be easily verified that in the case $V=0, G_{f}^{\sigma}(\overrightarrow{\mathrm{k}}, \omega)$ reduces to $G_{0}^{f \sigma}(\omega)$ (atomic limit) and $G_{d}^{\sigma}(\overrightarrow{\mathrm{k}}, \omega)$ to

$$
G_{d}^{\sigma}(\overrightarrow{\mathrm{k}}, \omega)=\frac{G_{0}^{d \sigma}(\omega)}{1-G_{0}^{d \sigma}\left(\epsilon_{\overrightarrow{\mathrm{k}}}-E_{B}\right)} .
$$

From Eqs. (10) we calculate the occupation numbers and the densities of states in the usual way.

In spite of the fact that the calculation can be performed in a rigorous self-consistent way, computing all mean values which appear in Eqs. (6), in this preliminary version we use the additional approximation

$$
\left\langle n_{i \bar{\sigma}}^{f \alpha} n_{i \sigma}^{d \beta} n_{i \bar{\sigma}}^{d \gamma}\right\rangle \simeq\left\langle n_{i \bar{\sigma}}^{f \alpha}\right\rangle\left\langle n_{i \bar{\sigma}}^{d \beta}\right\rangle\left\langle n_{i \bar{\sigma}}^{d \gamma}\right\rangle
$$

in order to simplify the numerical computations.

\section{RESULTS AND DISCUSSION}

We assume, to perform the calculation, a parabolic density of states for the unhybridized conduction band:

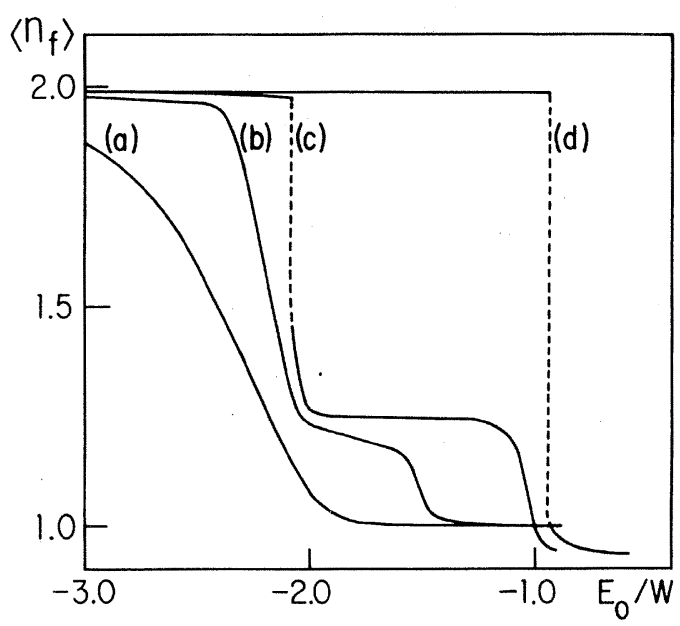

FIG. 1. Plot of $\left\langle n_{f}\right\rangle$ vs $E_{0}$, the energy of the $f$-level referred to $E_{B}=0$, with $V / W=0.2, U / W=2.0$, and $(a) G / W=0.0$; (b) $0.5,(c) 1.0$, and $(d) 1.5$.

$$
\rho_{d}(\epsilon)=\left\{\begin{array}{l}
\frac{3}{4 W}\left[1-\frac{\epsilon^{2}}{W^{2}}\right], \quad|\epsilon| \leq W \\
0, \quad|\epsilon|>W
\end{array}\right.
$$

The occupation number of the $f$ and $d$ shells are computed as a function of the relative position of $E_{0}$ and $E_{B}$. The variation of the parameter $\left(E_{0}-E_{B}\right) / W$ simulates the effect of the applied pressure. The Fermi level is determined by a total number of two electrons per atom. This guarantees, for $E_{0}+U \ll E_{B}-W$, a semiconducting state, as is the case for $\mathrm{Sm}$ monochalcogenides at normal pressure.

Two values of $V$ and four values of $G$ have been considered. In Fig. 1 we have plotted the occupation number of the $f$ shell as a function of $E_{0} / W$ (with $E_{B}=0$ ) with $V / W=0.2$ and $G / W$ equal to $(a) 0.0,(b) 0.5,(c) 1.0$, and (d) 1.5. It is evident, from the figure, that there exists a valence transition, continuous in the cases $a$ and $b$, and discontinuous in the cases $c$ and $d$. One can see also, an intermediate occupation of the $f$ level in the case $b$ and, more clearly, in $c$. This behavior corresponds, as we will see from the form of the densities of states, to the fact that when the conduction band begins to be filled, the residue of the pole of $G_{0}^{f \sigma}$ [Eq. (6a)] at $E_{0}+G$ is different from 0 . When $E_{0}+G$ enters the conduction band a second transition occurs to a value of $\left\langle n_{f}\right\rangle \sim 1$. The intermediate valence does not appear in the case $a$ because of $G=0$, and in $d$ because $E_{0}+G$ is too close to $E_{0}+U$, and inside the conduction band for $E_{0} \sim-1$.

In Fig. 2, a greater value of the hybridization is considered: $V / W=0.4$. In this case, with the same values of

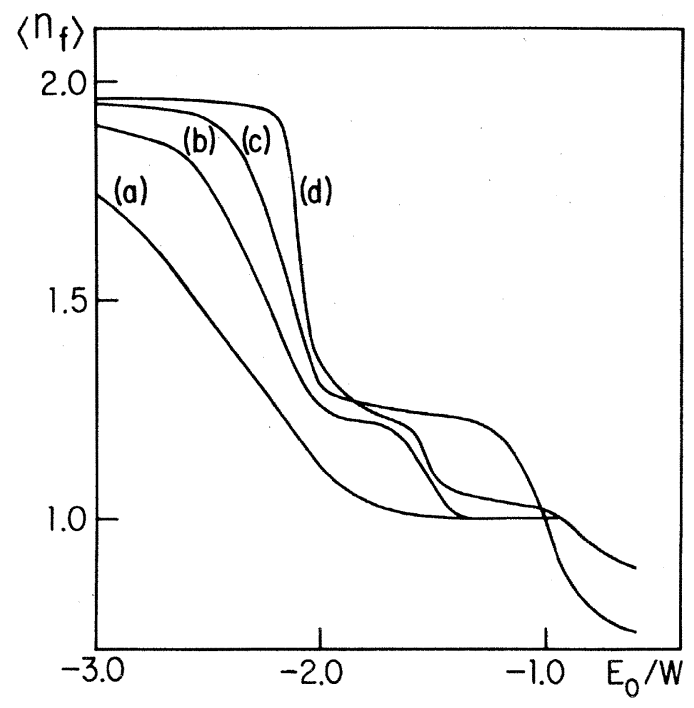

FIG. 2. Same as Fig. 1, with $V / W=0.4$. 


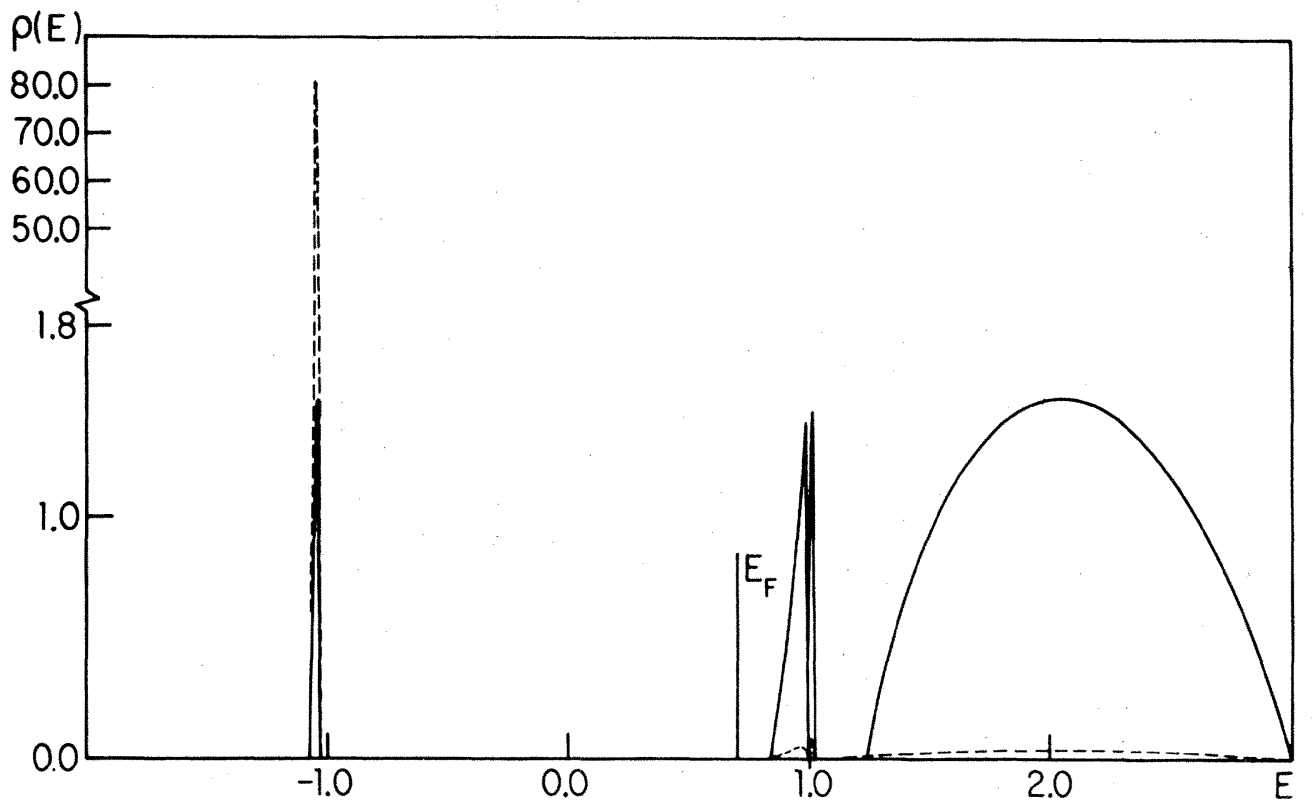

FIG. 3. Plot of the $d$ (solid line) and $f$ (dotted line) densities of states for $V / W=0.4, U / W=2.0$, and $G / W=1.0$, for $E_{0}=-3.0$.

$G$ the discontinuous transitions do not occur, but the intermediate valence is still present. This suggests that the type of transition is determined by the ratio $G / V$. One remarks that for $G / W=1$ and $1.5,\left\langle n_{f}\right\rangle$ does not stabilize on the value 1 , because the level $E_{0} \sim-1$ is strongly mixed with the conduction band.

It is also interesting to look at the plot of the densities of states. Because of the space available we have included here only three graphs, for the case $c$ of Fig. 2: $V / W=0.4, G / W=1$, and $E_{0}=-3.0$ (Fig. 3, before the transition), $E_{0}=-1.6$ (Fig. 4, the intermediate valence state) and $E_{0}=-1$ (Fig. 5, $\left\langle n_{f}\right\rangle \sim 1$ ).

Figure 3 shows the density of states for $E_{0}=-3$, that is, $\left\langle n_{f}\right\rangle \sim 2$. There is a peak of the $f$ density of states at $E_{0}+U \sim-1$. The $d$ states are separated in a main conduction band centered at $E_{B}+2 G=2$, and small peaks are centered at $E_{B}+G$ and $E_{0}+U$. Within the precision of the numerical computations, we have found the Fermi energy in the gap between $E_{0}+U$ and $E_{0}+G$; this result must be taken as an approximate one because it is well known that the Hubbard-I approximation leads to errors in the Fermi surfaces and to contradictions with the Luttinger theroem. ${ }^{14-16}$ (The Hubbard-I approximation should give an insulating phase only in the atomic limit for $\left\langle n_{f}\right\rangle=2$, that is, if $V=0$ and $\left.E_{0}+U \ll<E_{B}\right)$. On the other hand, calculations performed by using the alloy-

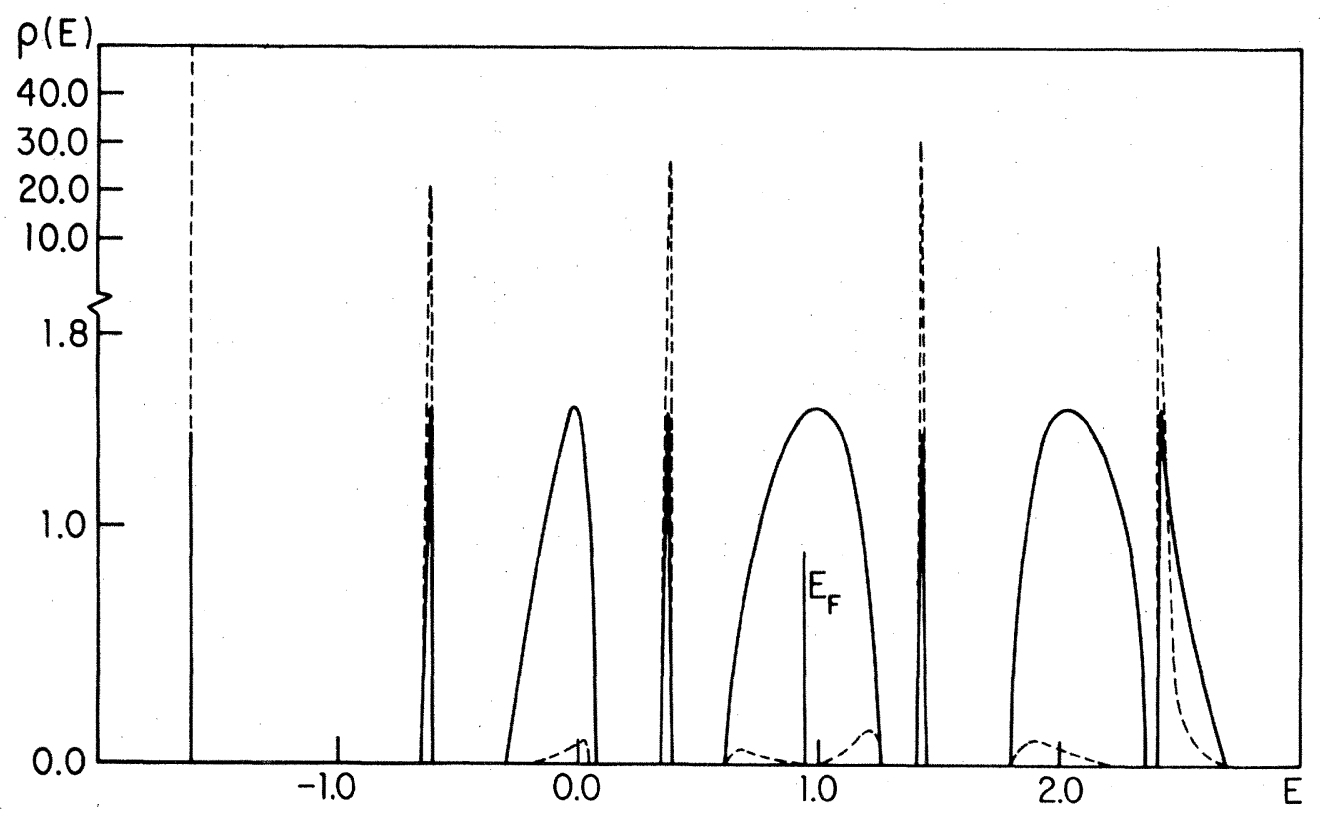

FIG. 4. Same as Fig. 3 for $E_{0}=-1.6$. 


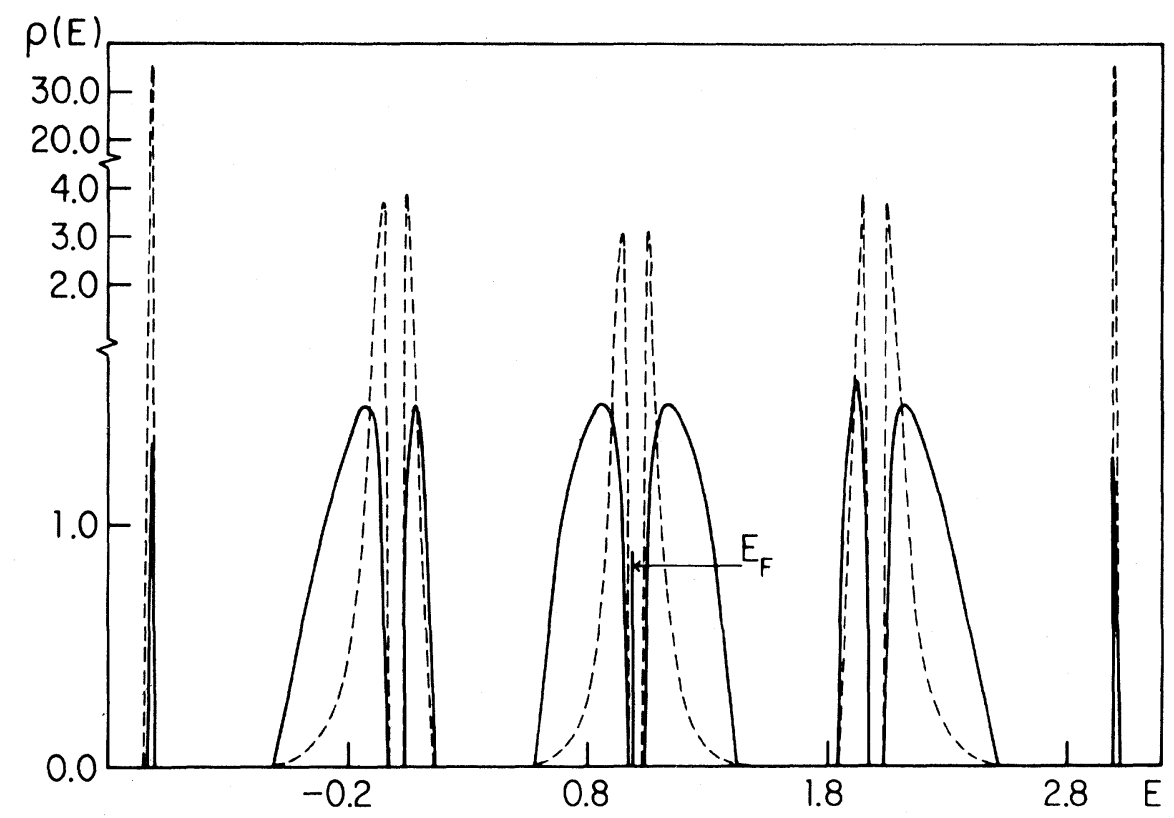

FIG. 5. Same as Fig. 3 for $E_{0}=-1.0$.

analogy approximation, as those performed by Martin and Allen, ${ }^{16}$ can give the correct insulating gap in this case, which corresponds to the "black" phase of SmS.

When $E_{0}=-1.6$, a fraction of the $f$ electrons in the level $E_{0}+U$ have been transferred to the conduction band, as is shown in Fig. 4. All the peaks of the $f$ density of states [Eq. (7a)] appear clearly, and $E_{0}$ and $E_{0}+G$ are below the conduction band, which is split into three subbands, centered at $E_{B}, E_{B}+G$, and $E_{B}+2 G$. The Fermi level is in the second conduction subband, and the system is a metal, in disagreement, again, with Luttinger theorem. $^{15}$

Finally, Fig. 5 exhibits the density of states for $\left\langle n_{f}\right\rangle \sim 1$ and $E_{0}=-1$. This is a special case, where there is electron-hole symmetry, and the Fermi level lies in a gap. This gap is due to the hybridization between the $f$ level at $E_{0}+U$ and the $d$ band at $E_{B}+G$. However, small changes of $E_{0}$ drive the Fermi level out of this gap.

One notices that the $f$ band is always separated into six narrow subbands, as in the atomic limit $(V=0)$. (However, with our choice for the numerical computation some levels coincide.) That splitting is certainly true because of the small value of $V$, the hybridization energy.

On the other hand, as our method is equivalent to the Hubbard-I approximation, ${ }^{13,17}$ for small $G$ the gaps between the conduction subbands at $E_{B}, E_{B}+G$, and $E_{B}+2 G$ could be induced by the approximation. ${ }^{14,17}$ Anyway, they are not essential to our description, since the semiconducting phase gap is of different nature. For the metal-insulator transition we must take care also, as was mentioned, since in many cases the Hubbard-I approximation does not give the correct Fermi surface. ${ }^{14} \mathrm{~A}$ discussion of this point can be found in Ref. 16 .

\section{CONCLUSIONS}

We applied here a full Hubbard-I treatment ${ }^{13,18}$ for a two-band Hamiltonian. This method reproduces the exact result in the atomic limit $\left(V=0, T_{i j}=T \delta_{i j}\right)$; it is reasonable to expect that the results are more accurate than in the Hartree-Fock theories previously utilized. ${ }^{2,5}$ In fact, an additional approximation was introduced, Eq. (12). However, we expect that the exact computation of the mean values which appear in Eq. (6) should not modify very much the present results. A full calculation, for $T=0$ and $T \neq 0$ is now in course.

Within this treatment we are able to obtain discontinuous transitions for $G \geq W$ and $V=0.2 W$, and continuous ones, for $V=0.4 W$. Also an intermediate value of the occupation number of the $f$ shell is found, and this value is stable over a wide range of values of the $f$ level energy, $E_{0}$. This result could explain the stable fractionary valence of some Sm compounds.

\section{ACKNOWLEDGMENTS}

This work was partially supported by Conselho Nacional de Pesquisas (CNPq) and Financiadora de Estudos e Projetos (FINEP), Brazil.
1J. M. Robinson, Phys. Rep. 51, 1 (1979).

${ }^{2}$ R. Ramírez, L. M. Falicov, and J. C. Kimball, Phys. Rev. B 2 , 3383 (1970); R. Ramírez and L. M. Falicov, ibid. $\underline{3}, 2425$ (1971).
${ }^{3}$ B. Coqblin and A. Blandin, Adv. Phys. 17, 281 (1968).

4J. R. Iglesias-Sicardi, A. K. Bhattacharjee, R. Jullien, and B. Coqblin, Solid State Commun. 16, 499 (1975); M. Avignon and S. K. Ghatak, ibid. 16, 1243 (1975). 
${ }^{5}$ C. E. T. Gonçalves da Silva and L. M. Falicov, Solid State Commun. 17, 1521 (1975).

${ }^{6}$ D. I. Khomskii and A. N. Kocharjan, Solid State Commun. 18,985 (1976).

${ }^{7}$ M. Plischke, Phys. Rev. Lett. $\underline{28}, 361$ (1972).

${ }^{8}$ D. K. Ghosh, Solid State Commun. 18, 1377 (1976).

${ }^{9}$ E. Baeck and G. Czycholl, Solid State Commun. 43, 89 (1982).

${ }^{10}$ K. Ueda, Solid State Commun. 41, 375 (1982).

$11 \mathrm{~J}$. R. Iglesias-Sicardi and I. Aveline, Solid State Commun. $\underline{36}$, 19 (1980); $\underline{37}, 794$ (1981).
${ }^{12}$ N. P. Montenegro and J. R. Iglesias-Sicardi, Physica 108B, 1357 (1981).

${ }^{13}$ E. V. Anda, J. Phys. C 14, L1037 (1981).

${ }^{14}$ W. Jones and N. H. March, Theoretical Solid State Physics (Wiley, London, 1973), Vol. 1, p. 341ff.

15 J. M. Luttinger, Phys. Rev. 119, 1153 (1960).

${ }^{16}$ R. M. Martin and J. M. Allen, J. Appl. Phys. 50, 7561 (1979).

17J. Hubbard, Proc. R. Soc. London, Sect. A 276, 238 (1963).

${ }^{18}$ M. A. Continentino and A. A. Gomes, Notas Fis. 20, 87 (1973). 